\title{
A Framework to Facilitate the Implementation of Technical Aspects of Web Accessibility
}

\author{
Roberto Cícero de Oliveira ${ }^{1}$, André Pimenta Freire ${ }^{2}$, Débora Maria Barroso Paiva ${ }^{1}$, \\ Maria Istela Cagnin ${ }^{1}$, and Hana Rubinsztejn ${ }^{1}$ \\ ${ }^{1}$ School of Computing, Federal University of Mato Grosso do Sul (UFMS) \\ P.O. Box 549, 79070-900, Campo Grande, MS, Brazil \\ \{rco.ufms, dmbpaiva, istela, hanaksr\} @gmail.com \\ ${ }^{2}$ Department of Computer Science, Federal University of Lavras (UFLA) \\ P.O. Box 3037, 37200-000, Lavras, MG, Brazil \\ apfreire@gmail.com
}

\begin{abstract}
The expansion of the internet has become apparent in recent years, both by the number of users, and by the number of services available on the network. Considering such an expansion it is essential that the content be accessible to all people, regardless their abilities or different disabilities. Thus, it is necessary that IT professionals dedicate time and effort in planning accessible online solutions. In this paper, we proposed the Homero framework in order to support the development of accessible interface layer of web applications. Developed using the PHP language, the Homero framework automates the generation of web pages in accordance with guidelines defined in Web Content Accessibility Guidelines (WCAG) 2.0. In order to provide evidence of the quality of web applications generated using the framework, an empirical study was conducted. The results showed the effectiveness of Homero to assist the development of accessible web applications, achieving level AAA in automatically detectable WCAG 2.0 success criteria.
\end{abstract}

Keywords: Web Accessibility, Framework, Empirical Study, Web Interfaces, WCAG.

\section{Introduction}

The expansion of the internet has become more and more apparent in recent years due to the number of users and the number of services available on the network, such as ebanking, e-learning and e-commerce.

Considering this expansion, it is necessary that professionals in Information Technology (IT) dedicate time and effort in planning intelligent and accessible online solutions, in order to enable all people to have access to information and web services. Based on the definition of accessibility provided by ISO 9241-171 - Ergonomics of Human-System Interaction Guidance on Software Accessibility - web accessibility could be defined as the possibility of a website to be used by people with widest range of abilities, including people with disabilities [1]. 
Additionally, the W3C (World Wide Web Consortium) published the WCAG (Web Content Accessibility Guidelines) [2], currently at version 2.0. The guidelines provide recommendations to help make the content of web pages more accessible to people with disabilities [2].

WCAG 2.0 consists of twelve accessibility guidelines, broken into 61 success criteria. These guidelines are organized around four principles (that content should be Perceivable, Operable, Understandable and Robust). In order to check the conformance to guidelines, developers can perform automatic evaluations of some success criteria, but manual evaluation is necessary to evaluate all criteria, including those that cannot be automatically evaluated. The completeness of automatic evaluation tools ranges between $14 \%$ and $38 \%$ for WCAG 2.0 success criteria [3].

However, developing accessible web applications is not always a trivial task, due to the knowledge needed to implement interface components in an accessible manner. One way to reduce the effort employed in the development of accessible web applications is using software reuse techniques such as software frameworks, which can contribute especially to the implementation of more technical accessibility requirements.

In this paper, we proposed the Homero framework in order to support the development of accessible interface layer of web applications. This framework automates the generation of web applications in accordance with the guidelines in WCAG 2.0, especially those which are related to more technical aspects, in order to reduce the effort spent by developers and the chance of occurring some common errors.

An empirical study was conducted to evaluate the number of violations of automatically detectable WCAG 2.0 success criteria in web applications generated using the framework. The study measured the number of elements implemented in the given time and the conformance level to WCAG 2.0 success criteria that can be evaluated automatically.

The paper is organized as follows. In Section 2, we discuss related work. In Section 3, the Homero framework is explained. In Section 4, the empirical study planning, execution and results are described. Finally, in Section 5 the conclusions are presented.

\section{Related Work}

Significant work has been dedicated to developing automated accessibility evaluation tools, such as Total Validator [4], CynthiaSays [5], iMergo [6] and others. Such automated evaluation tools can help developers detect many problems that otherwise would be very tedious to check. However, those tools cannot identify many problems that would require a manual evaluation. Even the problems that could be automatically detected often are identified in a late stage of the development, as developers do not always run such tools on their web pages as frequently as would be necessary. 
Other works in the literature have reported on different approaches to help develop more accessible content, including those that enable users and developers to embed Javascript codes into rendered web pages in their client browser and others that propose frameworks and model-based development approaches.

The scripting framework Accessmonkey [7] was developed in order to help end users and developers to incorporate Javascript code into web pages in order to make them more accessible. The framework allows for the inclusion of more general and specific codes in web pages in order to automate task that are commonly performed by users or to work around accessibility issues that are commonly encountered in web pages. However, the proposal of the framework is focused on the automation of page adaption in client-side operations.

Velasco [8] propose a framework to help develop more accessible rich internet applications (RIA) using a service-oriented architecture. The framework provides resources to help developers incorporate broader web-compliance requirements in the development of their projects. The framework allows for the use of resources to detect and adapt content according to web accessibility compliance requirements, especially for RIAs, which frequently have content that is dynamically generated after web pages were rendered.

Another proposal was made by Yan [9] by means of the SourceProbe tool, which embeds comments in HTML code of pages generated by server-side scripts. By means of those indications, when the final HTML code is evaluated using the client browser, it is possible to track where exactly in the server-side code the given part of resulting HTML with accessibility problems was generated.

Moreno et al. [10] proposed a Model-Driven Development (MDD) approach for modeling web accessibility and generating more accessible web applications. They developed the AWA - Accessibility for Web applications, which incorporates the AWA-Metamodel and a process for generating the code of web applications that follow basic principles of accessibility.

Martín et al. [11] proposed an approach based on Aspect-Oriented development to create more accessible web applications. Their approach consists of enriching User Interaction Diagrams with interaction points that incorporate accessibility requirements that can be modeled when the interface is designed. They use a Softgoal Interdependency Graph (SIG) template to include WCAG 1.0 requirements.

Although there are several mechanisms to help develop more accessible content, we could not find empirical studies in the literature that detailed the impact of using such approaches on adhering to the guidelines and on developers' productivity.

\section{The Homero Framework}

The Homero framework [12] automates the generation of user interfaces of web applications in accordance with a set of WCAG 2.0 guidelines, in order to reduce the effort spent by developers and the chance of occurring some common errors. One of 
the simplest examples of the framework usage is related to Success Criterion 1.1 in WCAG 2.0, which states: "Provide text alternatives for any non-text content so that it can be changed into other forms people need, such as large print, Braille, speech, symbols or simpler language". In this case, the framework can prevent developers from omitting alternative texts for images.

It should be noted that the Homero framework does not intend to address WCAG 2.0 success criteria that require manual human evaluation. For example, success criteria included in guideline 3.1 of WCAG 2.0 are related to "Making text content readable and understandable". The Homero framework is not able to determine whether content is completely readable and understandable automatically.

Homero is a black-box object-oriented framework [13], producing HTML 5.0 compliant code. It was developed using best practices in PHP development. The user interface layers of web applications generated by Homero are in accordance with success criteria of WCAG 2.0 AAA level that are related to technical aspects that can be checked automatically. Its classes were defined to prevent the overwriting of their methods, and thereby affecting the accessibility validations implemented.

By reusing Homero classes, developers implement the user interface layer of web applications using only PHP language. This enables the creation of clean and readable source code and reduces the cost of having to deal directly with HTML code, which is generated automatically by the framework.

The Homero framework has thirty-nine classes, organized into eleven groups to facilitate understanding. Each group consists of one or more classes, as shown in Fig. 1.

The Media group is responsible for media of user interface (audio and video). The objects group represents external components (i.e. flash plug-in) which may be incorporated in the interface. The Link group deals with links or anchors of the interface. The Images group treats the images that are part of the application context. The Layout group works with the divisions of the site layout. The application tables are handled by Tables group. The Lists group was created to deal with lists. The Texts group consists of ten classes and works with different types of texts. The Form group is responsible for managing forms. The Header group deals with header of HTML pages. The Page group represents the web page to be developed.

The Homero instantiation must begin by creating an object of Page class. This object has a header attribute, that accepts an object of the Header class, and a set of objects of Element class, which are instances of other classes (such as Media, Object, Link, Image, Table, Superscript, etc), according to the inheritance relationships with the Element class, illustrated in Fig. 1.

During the methods invocation of Homero classes, the developer must provide as parameters all required accessibility data. Otherwise, an accessibility validation warning of the user interface of the web application is presented, as can be seen in the warning shown on the right side of Fig. 2. In this case, the alternative text of the image represented by img object of Image class (line 9, Fig. 2) has not been defined, because the second parameter of the constructor method was defined as null, causing an accessibility error. 


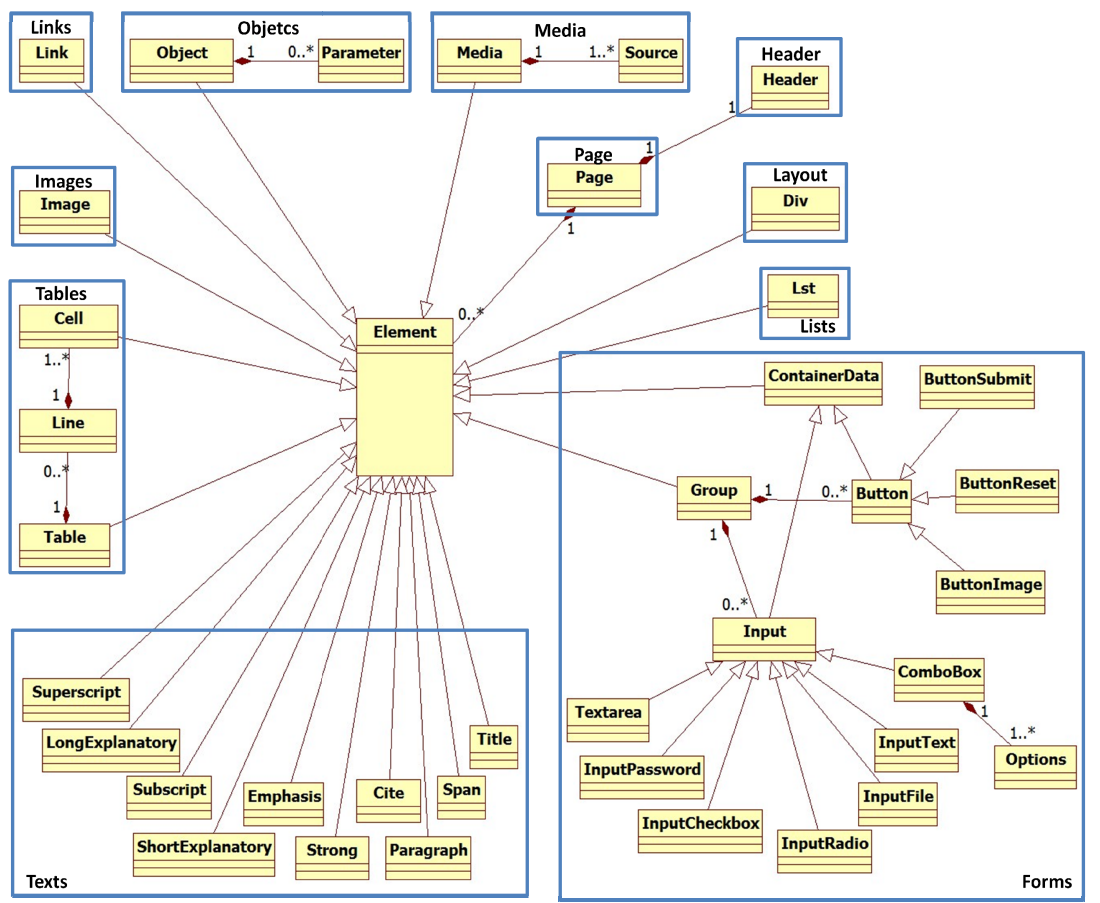

Fig. 1. Homero class diagram

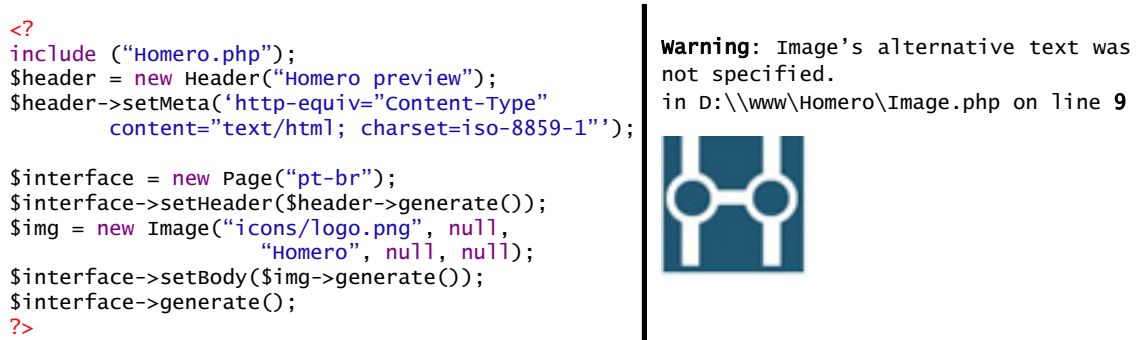

Warning: Image's alternative text was not specified. in $\mathrm{D}: \backslash \backslash w w w \backslash$ Homero $\backslash$ Image.php on line 9

Fig. 2. Example of the Homero instantiation

For the Homero instantiation, a property of the PHP language is employed. In order to use classes, it is necessary only to include them in the source code with the following PHP native functions: include or include_once. Thus, a file called Homero.php was created with all framework classes, which must be included in all project files.

Initially, it is just necessary to include the Homero.php file in the file where the user interface will be created (line 2, Fig. 2) and to add the folder that contains the files of framework classes in the folder of the web application project. 
Every file of the user interface layer of the web application must have an object of the Page class (line 7, Fig. 2). Then, the objects of Homero classes necessary to compose the user interface should be created - for example, line 3 (header object) and line 9 (img object) of Fig. 2.

Finally, to generate the user interface it is necessary to invoke the generate () method of object of Page class (line 12, Fig. 2).

\section{$4 \quad$ Empirical Study}

\subsection{Empirical Study Planning}

An empirical study was planned according to Wholin et al. [14] to evaluate the number of violations of automatically detectable WCAG 2.0 success criteria in web applications generated using the Homero framework. The study had the participation of $25 \mathrm{MSc}$ students, divided into eleven development teams with similar knowledge about accessibility. These teams were divided into two groups (one using Homero - GH and another not using it - $\mathrm{GnH}$ ) and were asked to develop a web application interface according to the same specification. We evaluated the number of elements implemented in the given time and the conformance level of WCAG 2.0 success criteria that can be evaluated automatically. The latter was obtained using the Total Validator tool [4].

As shown in Table 1, two hypotheses and metrics were defined to analyze the effect of the use of Homero. The metric related to the first hypothesis was the number of elements implemented in the layout of the user interface. For the second hypothesis, the metric was the level of accessibility achieved by the application, considering WCAG 2.0 (A, AA or AAA), using Total Validator tool.

Table 1. Hypotheses and metrics of the empirical study

\begin{tabular}{|c|l|}
\hline $\mathrm{H}_{0}$ & $\begin{array}{l}\text { There is no difference in the number of elements developed using, or } \\
\text { not, Homero }\end{array}$ \\
\hline $\mathrm{H}_{\mathrm{a} 0}$ & $\begin{array}{l}\text { There is a significant difference in the number of elements developed } \\
\text { using, or not, Homero. }\end{array}$ \\
\hline Metric & \multicolumn{1}{c|}{ Number of elements implemented in the layout } \\
\hline \multicolumn{2}{|c|}{} \\
\hline $\mathrm{H}_{1}$ & $\begin{array}{l}\text { There is no difference in level of accessibility achieved by using or not } \\
\text { Homero. }\end{array}$ \\
\hline $\mathrm{H}_{\mathrm{a} 1}$ & $\begin{array}{l}\text { There is a significant difference in the level of accessibility achieved } \\
\text { by using or not Homero. }\end{array}$ \\
\hline Metric & \multicolumn{2}{c}{ Level of accessibility in WCAG 2.0 } \\
\hline \multicolumn{2}{|c|}{ Legend: $\mathrm{H}$ - Null hypothesis, $\mathrm{H}_{\mathrm{a}}$ - Alternative hypothesis } \\
\hline
\end{tabular}

Before conducting the empirical study, a survey was conducted to analyze the level of knowledge, theoretical and practical, of the participants in object-oriented programming, web development using PHP and HTML languages. Moreover, the level 
of experience of the participants with concepts of web accessibility and WCAG 2.0 guidelines was identified. This survey was conducted through a questionnaire answered by the participants and the level of knowledge of participants is presented in Table 2.

Table 2. Level of Experience of Participants

\begin{tabular}{|l|r|r|r|r|}
\hline $\begin{array}{c}\text { Level of theoretical } \\
\text { knowledge }\end{array}$ & \multicolumn{1}{c|}{ None } & \multicolumn{1}{c|}{ Little } & Reasonable & \multicolumn{1}{c|}{ High } \\
\hline Object-oriented programming & 0 & 6 & 16 & 3 \\
\hline PHP & 3 & 11 & 7 & 4 \\
\hline HTML & 1 & 9 & 11 & 4 \\
\hline Web Accessibility & 5 & 14 & 6 & 0 \\
\hline WCAG 2.0 guidelines & 22 & 3 & 0 & 0 \\
\hline
\end{tabular}

Considering the results in Table 2, a training was offered to groups about object oriented programming, PHP language, Homero framework, tableless development, HTML language and WCAG 2.0.

Participants were organized into teams of 2 or 3 people. To allow comparison between development using Homero and development without the use of Homero, teams were divided into two groups: one group, called GH, used Homero in the development and the other, called $\mathrm{GnH}$, which did not use Homero. The formation of teams and groups was made so that each team in each group had equivalent knowledge in relation to technologies used for developing activities.

The execution plan of the study was composed of a single phase, in which the teams from the two groups had to develop the proposed interface layout. At the beginning of the study we provided the teams with the interface model to be developed, a consent form to use the information and an activities execution form to assess the framework usage. The participants were provided with:

1. A mockup of a semantically accessible application, used as template of the interface;

2. An image of the expected appearance of the interface, with the divisions of the proposed layout;

3. A CSS file containing the style characteristics of the interface, and

4. A folder containing five images used in the interface.

\subsection{Execution and Results of the Empirical Study}

After the execution of the activities assigned to the participants, some results were analyzed as shown below.

During the evaluation of the hypothesis 1 it was observed that the average number of elements implemented by teams of $\mathrm{GnH}$ group was higher (9.6 versus 6.2 of $\mathrm{GnH}$ group), as can be seen in Fig. 3 . 


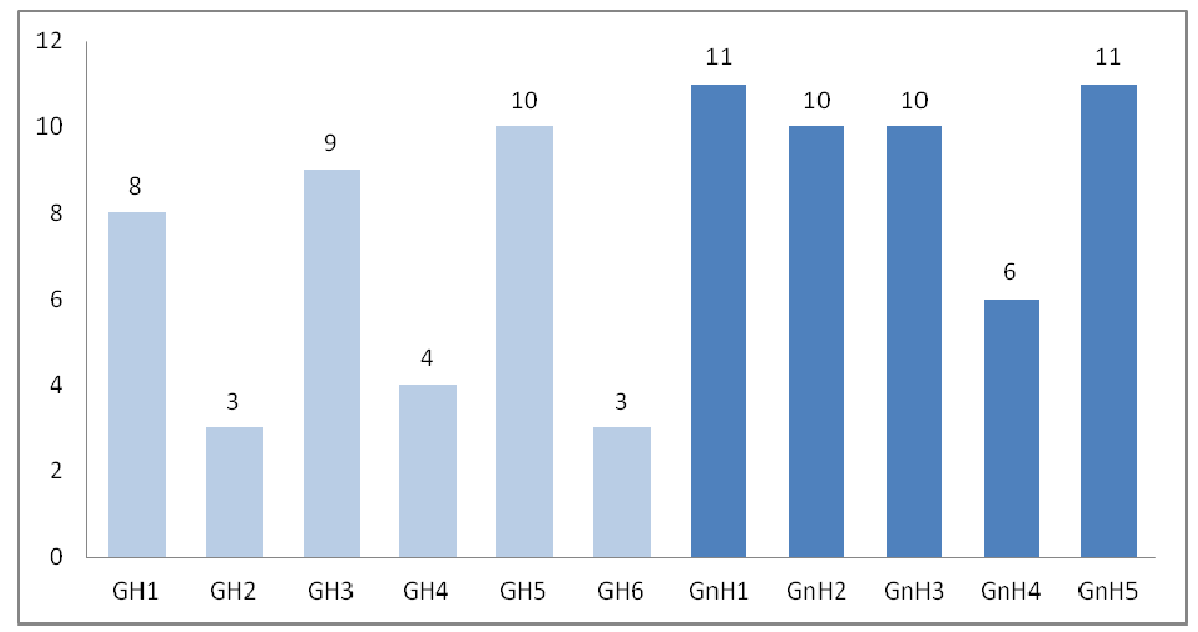

Fig. 3. Number of elements implemented by teams

As observed, half of GH teams could implement few elements. We believe that this discrepancy may be due to lack of knowledge of Homero framework. In the form of activities execution of the $\mathrm{GH}$ group, it was observed that this was the main difficulty ( $50 \%$ of the teams indicated this); despite the Homero training had been offered before the empirical study execution.

To evaluate the hypothesis 2 , the source files developed by participants were validated using the Total Validator tool. The result of this validation is the number of errors of accessibility automatically evaluated in each user interface (Fig. 4) and the level of WCAG 2.0 reached (Fig. 5). On the y-axis of Fig 5, number 1 corresponds to level A of WCAG 2.0, number 2 corresponds to level AA and number 3 corresponds to the level AAA.

The results showed that teams of $\mathrm{GnH}$ group developed interfaces with a larger number of elements. However, all of them presented accessibility errors. In contrast, all interfaces developed by the GH group teams, even with a smaller number of elements implemented, had no violations of automatically detectable WCAG 2.0 success criteria of AAA level.

Therefore, we concluded that the use of Homero framework may help avoid developers running into common accessibility mistakes that can be detected automatically earlier in the development, saving time and effort that can be dedicated to performing manual evaluations and user testing. Additionally, Homero contributes to widening the use of Web accessibility guidelines and to making accessibility a concern throughout the development of web applications. 


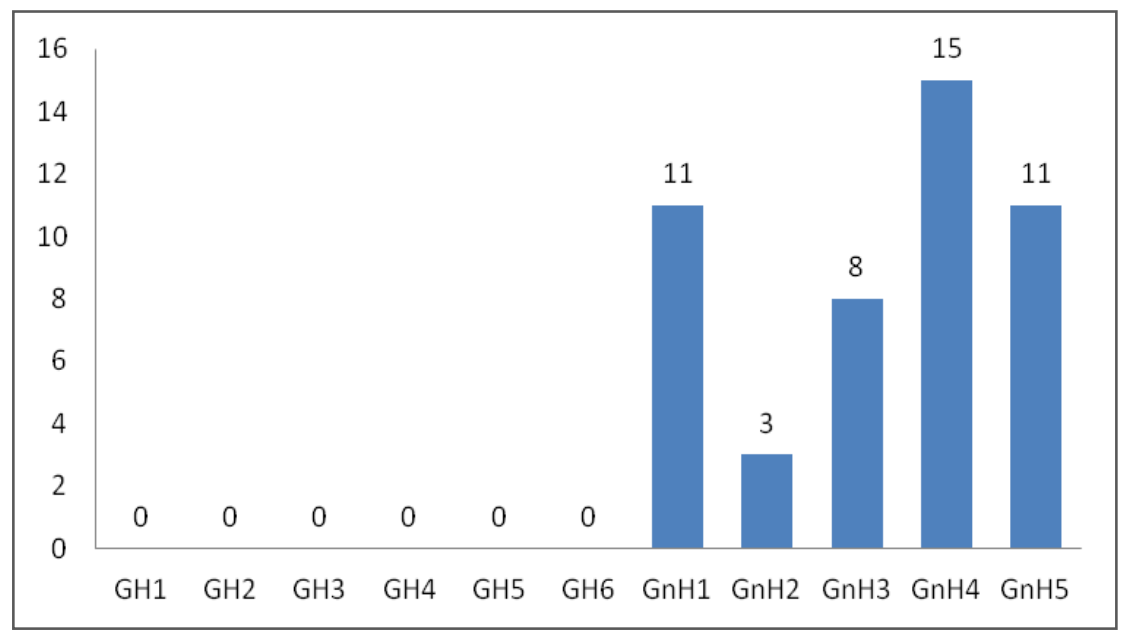

Fig. 4. Number of errors of accessibility by groups

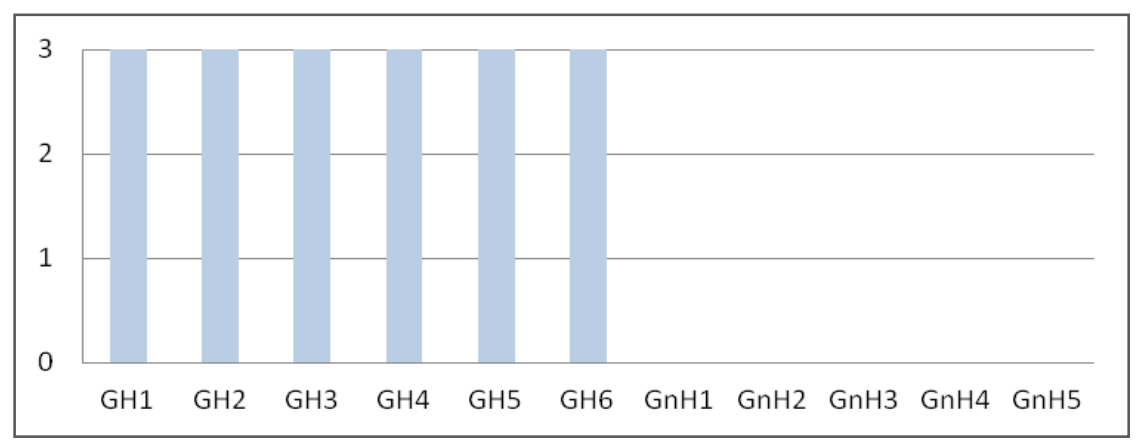

Fig. 5. WCAG 2.0 conformance level achieved by groups

\section{Conclusion}

This paper presented a study consisting of the development of a framework to help develop more accessible web applications with easier implementation of accessibility requirements from guidelines that can be automatically detected.

Along with the proposal of the framework, an empirical study with 25 participants was conducted to verify the extent to which the framework helped implement technical requirements of accessibility and the productivity of developers using the framework or not.

The initial results from this first study showed that, despite lowering the productivity of developers who used the framework for the first time, the number of technical accessibility errors in the generated web pages is substantially reduced. 
This shows that the use of frameworks and other approaches to software reuse can be an important ally to help improve the process of producing more accessible web applications.

As future work, we intend to perform further studies to analyze whether the use of the framework can also help developers have greater concern about accessibility. We also aim to investigate whether this would have an impact on reducing the number of accessibility problems encountered in manual accessibility inspections by experts and by testing with disabled users.

We also intend to investigate whether developers can be more productive using the framework after having had more experience using it. Finally, we intend to integrate the use of the framework into a broader proposal for a development process that takes into account a more diverse set of accessibility requirements, especially in the phase of code generation.

Acknowledgements. Financial support for this study was provided by Fundect T.O. 0039/11, Capes and PET Project SESU/MEC/FNDE.

\section{References}

1. International Standards Organization: ISO 9241-171: Ergonomics of Human-System Interaction. Part 171: Guidance on software accessibility (2008)

2. Caldwell, B., Cooper, M., Reid, L.G., Vanderheiden, G.: Web Content Accessibility Guidelines 2.0. Web Accessibility Initiative (WAI), World Wide Web Consortium (W3C) (2008), http: / / www .w3 . org/TR/WCAG2 0 (accessed October 23, 2013)

3. Vigo, M., Brown, J., Conway, V.: Benchmarking Web Accessibility Evaluation Tools: Measuring the Harm of Sole Reliance on Automated Tests. In: 10th International CrossDisciplinary Conference on Web Accessibility (W4A 2013), pp. 1-10. ACM, New York (2013)

4. Total Validator - accessibility evaluation tool (2014), http: / / www . totalvalidator. com/ (last accessed February 4, 2014)

5. Hisoftware. CynthiaSays - accessibility evaluation tool (2014), http: / / www . cynthiasays . com (last accessed February 4, 2014)

6. Mohamad, Y., Stegemann, D., Koch, J., Velasco, C.A.: Imergo: Supporting Accessibility and Web Standards to Meet the Needs of the Industry via Process-Oriented Software Tools. In: Miesenberger, K., Klaus, J., Zagler, W.L., Burger, D. (eds.) ICCHP 2004. LNCS, vol. 3118, pp. 310-316. Springer, Heidelberg (2004)

7. Bigham, J.P., Ladner, R.E.: Accessmonkey: A Collaborative Scripting Framework for Web Users and Developers. In: 2007 International Cross-Disciplinary Conference on Web Accessibility (W4A 2007), pp. 25-34. ACM, New York (2007)

8. Velasco, C.A., Denev, D., Stegemann, D., Mohamad, Y.: A Web Compliance Engineering Framework to Support the Development of Accessible Rich Internet Applications. In: 2008 International Cross-Disciplinary Conference on Web Accessibility (W4A 2008), pp. 45-49. ACM, New York (2008)

9. Yan, S.: SourceProbe: Web Accessibility Remediation Framework. In: 2010 International Cross Disciplinary Conference on Web Accessibility (W4A 2010), article 26, 2 pages. ACM, New York (2010) 
10. Moreno, L., Martínez, P., Ruiz, B.: A MDD Approach for Modeling Web Accessibility. In: 7th Int. Workshop on Web-Oriented Software Technologies, pp. 1-6 (2008)

11. Martín, A., Rossi, G., Cechich, A., Gordillo, S.: Engineering Accessible Web Applications. An Aspect-Oriented Approach. World Wide Web 13(4), 419-440 (2010)

12. Oliveira, R.C.: Homero: A Framework for Developing Accessible User Interface of Web Applications. Master Thesis, College of Computing, Federal University of Mato Grosso do Sul (2013) (in Portuguese)

13. Fayad, M.E., Johnson, R.E.: Domain-specific Application Frameworks: Frameworks experience by industry, 1st edn. John Wiley \& Sons (2000)

14. Wohlin, C., Runeson, P., Höst, M., Ohlsson, M.C., Regnell, B., Wesslén, A.: Experimentation in Software Engineering. Springer (2012) 